\title{
Demonstration of a Shaped Beam Reflectarray Using Aperture-Coupled Delay Lines for LMDS Central Station Antenna
}

\author{
Eduardo Carrasco, Member, IEEE, Manuel Arrebola, Member, IEEE, José A. Encinar, Member, IEEE, and \\ Mariano Barba
}

\begin{abstract}
A shaped-beam reflectarray based on patches, aperture-coupled to delay lines is demonstrated for local multipoint distribution system (LMDS) central station antennas, in the $10.10-10.70 \mathrm{GHz}$ band. The antenna must cover a $60^{\circ}$-sector in azimuth with a squared cosecant pattern in elevation. The design process consists of two steps. First, a phase-only pattern synthesis technique is applied to obtain the required phase-shift distribution on the reflectarray surface which generates the shaped pattern. The second stage consists of determining the length of the delay lines, aperture-coupled to the square patches, in order to achieve the phase distribution synthesized in the previous step. Two reflectarray antennas have been designed, one for vertical (V) and the other for horizontal $(\mathrm{H})$ polarization. A breadboard for $V$-polarization has been manufactured and tested in an anechoic chamber, showing a good agreement between theoretical and measured radiation patterns.
\end{abstract}

Index Terms-Aperture coupling, local multipoint distribution system (LMDS), reflectarray, shaped beam antennas.

\section{INTRODUCTION}

A local multipoint distribution system (LMDS) is a broadband wireless access technology that provides a point-tomultipoint microwave connection in those areas where cable- or fiber-based networks are too expensive or inappropriate because of the paucity of customers or geographical conditions. The requirements for the LMDS base-station antennas have been defined by the European Telecommunications Standards Institute (ETSI). A sectored beam in azimuth and a squared cosecant shape in elevation are specified for central station antennas [1]. The European Conference of Postal and Telecommunications Administrations (CEPT) allocates the paired bands 10.15-10.30 GHz and $10.50-10.65 \mathrm{GHz}$ for fixed systems including point to multipoint [2].

A reflectarray is a low-profile planar antenna made up of a reflective array of printed patches, see Fig. 1, with a certain tuning on the phase of the reflected wave to produce a focused

Manuscript received December 11, 2007; revised June 13, 2008. Current version published October 3, 2008. This work was supported in part by the Spanish Ministry of Science and Technology under the MEC TEC 2004-02155 project and by the Mexican National Council of Science and Technology (CONACYT).

E. Carrasco, J. A. Encinar, and M. Barba are with the Electromagnetism and Circuit Theory Department, Universidad Politecnica de Madrid, 28040 Madrid, Spain (e-mail: carrasco@etc.upm.es).

M. Arrebola is with the Electrical Engineering Department, Universidad de Oviedo, 33203 Gijon (Asturias), Spain.

Digital Object Identifier 10.1109/TAP.2008.929452

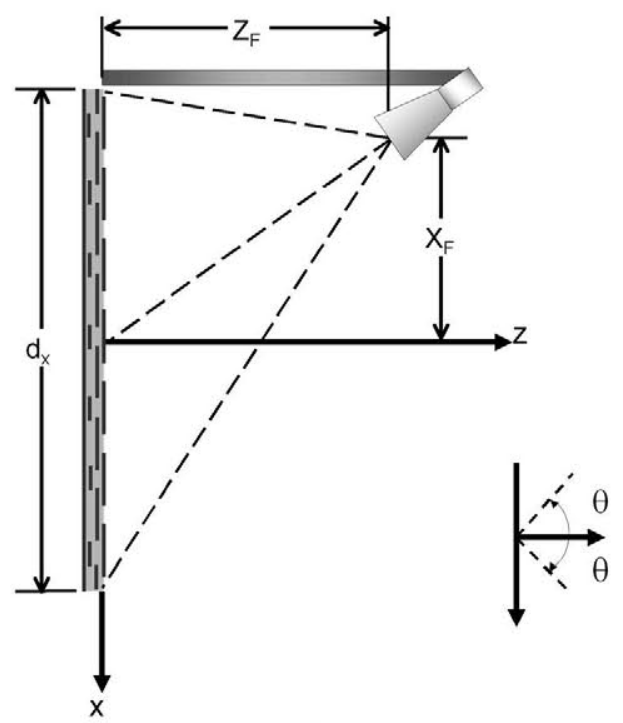

(a)

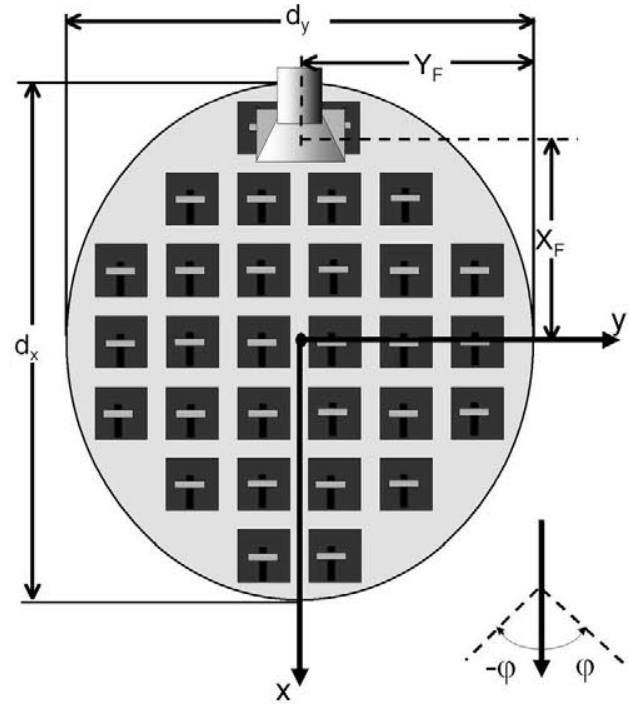

(b)

Fig. 1. Drawing of a generic reflectarray antenna (a) lateral view and (b) upper view.

or shaped beam when illuminated by a primary feed. The reflectarray is manufactured on a flat panel, without the need for molds as in reflector antennas. As a result, the manufacturing process and cost are the same to generate a focused or shaped beam. 
Reflectarrays based on varying-sized rectangular patches have been used to shape the beam for space and LMDS applications [3]-[5]. Rectangular patches coupled through a rectangular aperture to delay lines of variable length, have been used for single or dual linear polarization reflectarrays [6], [7] to generate pencil beams. The use of these elements in shaped-beam reflectarrays offers several advantages over other configurations. First, by printing the delay lines on a different layer than the radiating elements, there is more room for the inclusion of control switches such as PIN diodes [8] or micro electromechanical systems (MEMS) which permit the beam reconfiguration [9]. Besides, the effect of the differential spatial phase delay [10] is reduced through the introduction of a physical path that produces true-time delay (TTD) without phase limitation [11]. This compensation avoids the reduction in gain for pencil-beam reflectarrays [12] and the shape distortion for contoured beam reflectarrays [3], especially in large antennas. Reflectarray elements based on aperture-coupled patches with lines of variable length have previously been designed, manufactured and measured, showing a wide range of phase delay, low reflection losses and good element bandwidth [11]. The validation of the aperture-coupled elements was made by measuring the amplitude and phase of the reflection coefficient of two elements within a WR-90 waveguide, using the well-known waveguide simulator technique (WGS) [11]. A very linear response of the phase-shift as a function of the line length can be achieved when the geometrical parameters are adjusted correctly [13].

In this paper, two circular reflectarrays based on patches, aperture-coupled to delay lines have been designed to produce the required shaped beam in the $10.10-10.70 \mathrm{GHz}$ band for an LMDS central station, considering vertical (V) and horizontal (H) polarization, respectively. Although only a $6 \%$ bandwidth is required in the present application, one advantage of the proposed aperture-coupled configuration is that it provides good bandwidth performances. A breadboard for V-polarization has been manufactured and tested in an anechoic chamber with acceptable results.

\section{REFLECTARRAY DESIGN}

For the design of a contoured-beam reflectarray, a direct optimization process, in which the delay lines are adjusted in an iterative process to achieve the required contoured pattern, is computationally unaffordable, because it would require the analysis of all the elements (several hundreds) to compute the radiation pattern in each iteration of the pattern synthesis. Then, an efficient alternative that consists of dividing the design process into two steps as described in [3] has been used. In the first step, assuming a fixed amplitude distribution on the reflectarray surface given by the feed radiation pattern, a phase-only synthesis is applied to compute the phase of the reflected field at each reflectarray element that provides the required contoured pattern. In the second step, the lengths of the delay line are adjusted element by element to implement the previous phase distribution.

In the pattern synthesis, the reflectarray elements are considered ideal, neglecting the losses, and the polarization coupling, therefore only co-polar patterns are considered in the pattern synthesis. This assumption is made only for obtaining the required phase distribution in the reflectarray, but when the delay lines are adjusted, a full-wave based on the method of moments (MoM) [14] is used to analyze the reflectarray elements. If necessary, the cross-polarization of the elements can also be taken into account in the pattern synthesis technique, as described in [15]. However the assumption of ideal phase-shifters for the synthesis is accurate when the losses and cross-polarization in the reflectarray element are very low, as is the case of the proposed aperture-couple element. used in this work. This technique has been validated by a very good agreement between simulated and measured radiation patterns in a three-layer reflectarray made of varying-sized patches [4].

\section{A. Phase-Only Pattern Synthesis}

The radiation pattern must be a squared cosecant in elevation and sectored in azimuth. As the sector angle is not imposed and can vary between $15^{\circ}$ and $180^{\circ}$, a sector beam covering $60^{\circ}$ has been chosen. The ETSI specifies that the antenna gain should be greater than $16.25 \mathrm{~dB}$ for a $60^{\circ}$-sector, and also defines a minimum gain template in elevation. However, a maximum gain template is also defined for the pattern synthesis. The minimum and maximum gain templates in the main planes are shown in Fig. 2 for both elevation and azimuth planes. The three-dimensional masks are obtained as the combination of both masks:

$$
\mathrm{M}_{1}(\mathrm{u}), \mathrm{M}_{2}(\mathrm{v}) \rightarrow \mathrm{M}(\mathrm{u}, \mathrm{v})
$$

where $u=\sin (\theta) \cos (\varphi)$ and $v=\sin (\theta) \sin (\varphi)$. The difference between maximum and minimum gain in the coverage region has been fixed as $2 \mathrm{~dB}$ and the tilt angle is $11^{\circ}$. This angle is defined as a fixed, angular shift of the reflectarray main beam axis (boresight) in the elevation plane which is used to achieve the required coverage area. The elevation template defines the mask in the $u$ plane and the azimuth defines the mask in the $\mathrm{v}$ plane. By combining the two templates, the maximum and minimum three-dimensional masks are generated. Fig. 3 shows the minimum and maximum 3-D masks.

The amplitude of the field on the reflectarray surface is imposed by the radiation pattern of the feed, assuming that the reflectarray elements are in the far field region of the feed source. Then, only the phase can be adjusted to shape the beam, which means that a phase-only pattern synthesis technique must be applied. A commercial Narda-640 feed horn has been used to feed the reflectarray, and the far field has been modeled as a $\cos ^{\mathrm{q}}$ function in order to determine the illumination levels on the reflectarray surface. The q value has been calculated to match the radiation pattern of the horn at the design frequency $(10.4 \mathrm{GHz})$.

The choice of the starting point in the synthesis process is critical when the number of variables is large enough as in reflectarrays. An approximate phase distribution that will be used as starting point is obtained by combining two linear phase distributions corresponding to the azimuth and elevation patterns. The one-dimensional phase distributions for a sector and cosecant squared are obtained independently using an analytic phase-only synthesis technique, as described in [16]. The 2-D phase distribution obtained by combining the previous phases 


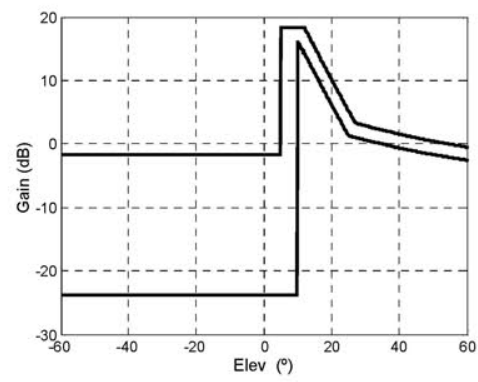

(a)

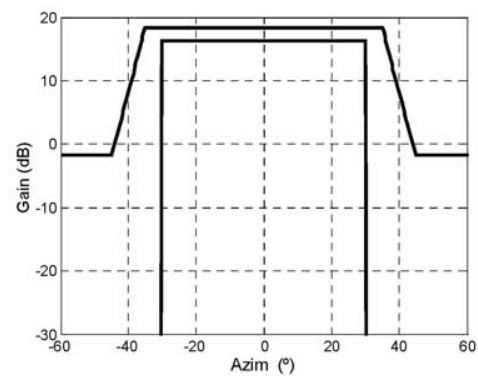

(b)

Fig. 2. Specification templates (a) elevation (plane $\varphi=0^{\circ}$ ) and (b) azimuth (plane $\varphi=90^{\circ}$ ).

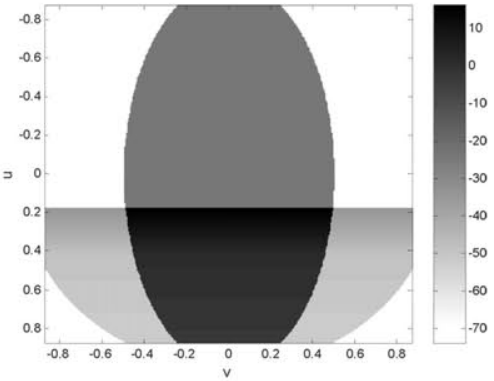

(a)

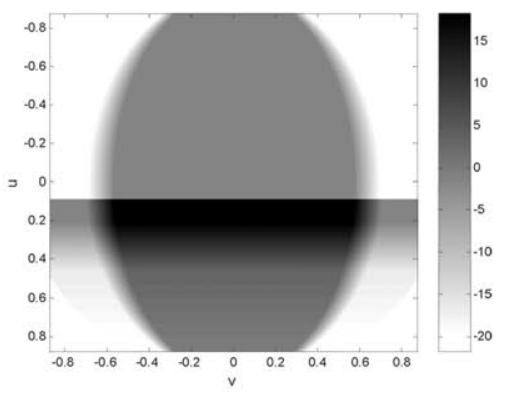

(b)

Fig. 3. 3-D masks for (a) minimum and (b) maximum gain.

is used as starting point in a two-dimensional pattern synthesis based on the Intersection Approach technique described in [17].

Taking into account the amplitude distribution provided by the horn, the phase distribution on the reflectarray surface has been synthesized for the geometrical parameters defined in Table I, and the result is shown in Fig. 4. This distribution is valid for the two linear polarizations $(\mathrm{H}$ and $\mathrm{V})$, which can

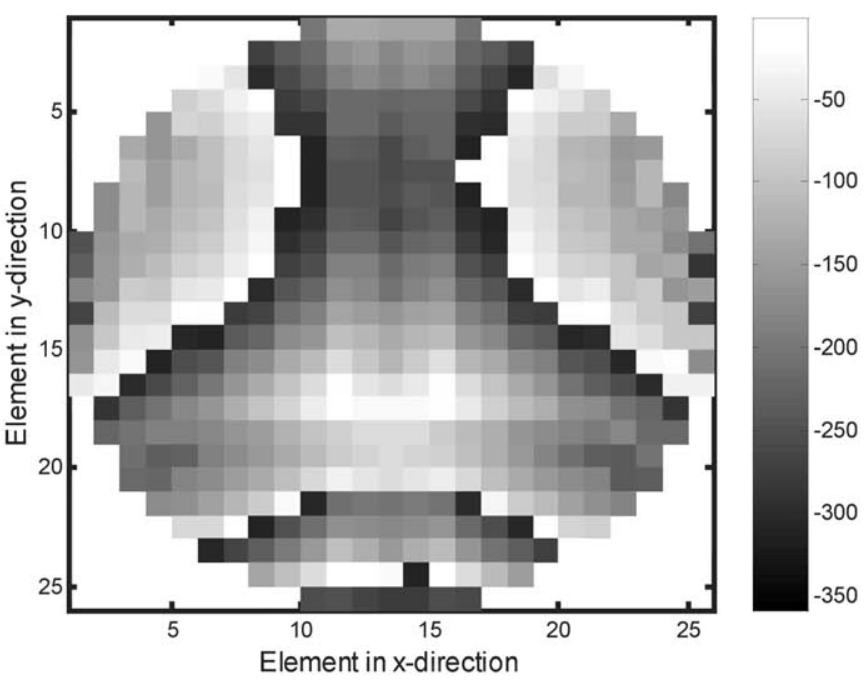

Fig. 4. Synthesized phase distribution of the reflection coefficient to generate the required shaped beam.

TABLE I

REFLECTARRAY DATA

\begin{tabular}{c|cc}
\hline \multicolumn{2}{c}{ Reflectarray Diameter $(\mathrm{mm})$} & $412.50 \mathrm{~mm}$ \\
\hline \multicolumn{2}{c}{ Number of elements } & 489 \\
\hline \multicolumn{2}{c}{ Period } & 16.5 \\
\hline \multirow{3}{*}{$\begin{array}{c}\text { Feed } \\
\text { position }\end{array}$} & $\mathrm{X}(\mathrm{mm})$ & -175 \\
\cline { 2 - 3 } & $\mathrm{Y}(\mathrm{mm})$ & 0 \\
\cline { 2 - 3 } Design frequency $(\mathrm{GHz})$ & 390 \\
\hline
\end{tabular}

be implemented by the proposed reflectarray element if it is rotated by $90^{\circ}$.

\section{B. Delay Line Implementation}

In the second step, the lengths of the delay lines of the reflectarray element shown in Fig. 5 are determined. The unit cell consists of a fixed-sized square patch coupled through a fixed-sized rectangular slot to a variable length delay line. The length of the line is accurately adjusted in each element, through an iterative process that calls an analysis routine, taking into account the angle of incidence. The analysis routine is based on the MoM in spectral domain for multilayer periodic structures [14], and takes into account the dissipative losses in the dielectric layers and the cross-polarization generated by the reflectarray elements. The incident field coming from the feed at an angle of incidence $(\theta, \varphi)$ is considered as the sum of two orthogonal components, parallel to the rectangular lattice of the reflectarray. Then, the two components of the field reflected (and scattered) by each element are computed. The mutual coupling between elements is automatically computed by the assumption of local periodicity, where each element is analyzed in a periodic environment. This approach is very accurate for the analysis of the proposed reflectarray configuration, because the patches and the apertures are identical for all the elements, where the length of the lines is the only parameter that changes.

The reflectarray element has been designed by properly adjusting all of the geometrical parameters in order to provide a linear phase response, a good element bandwidth and low losses [13]. The multilayer configuration is shown in Fig. 5, and the 


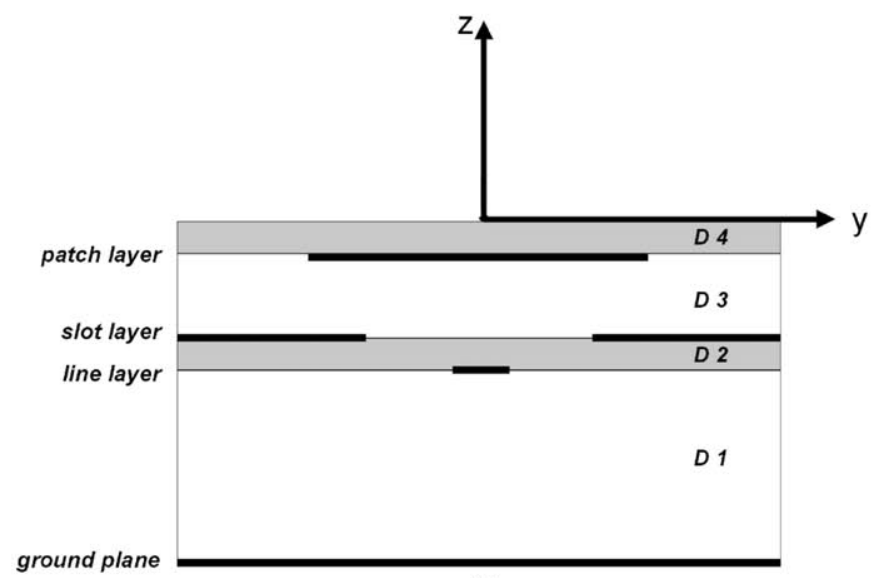

(a)

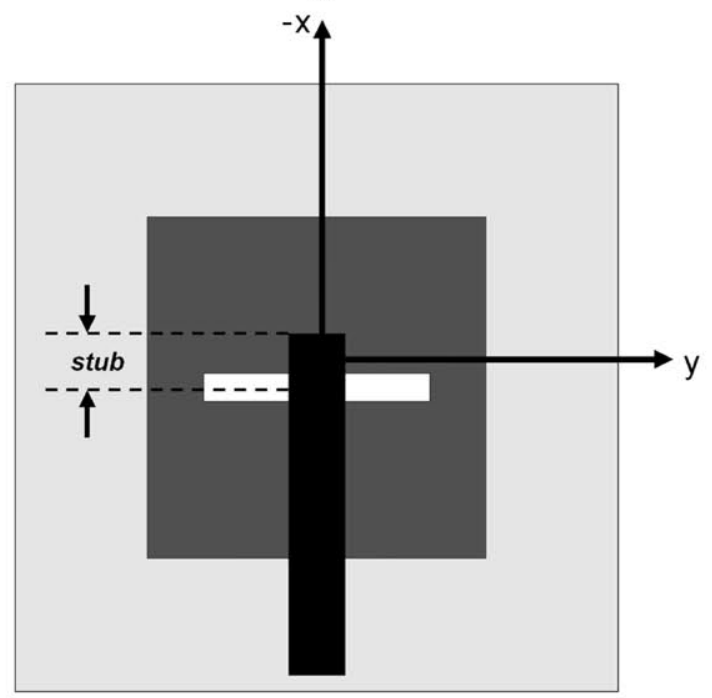

(b)

Fig. 5. A patch, aperture-coupled to a delay line, used as phase-shifter element (a) side and (b) top view.

TABLE II

DEFINITION OF REFLECTARRAY ELEMENT

\begin{tabular}{cccc}
\hline Layer & $\mathrm{X}(\mathrm{mm})$ & \multicolumn{2}{c}{$\mathrm{Y}(\mathrm{mm})$} \\
\hline Period & 16.50 & \multicolumn{2}{c}{16.50} \\
\hline Patch & 9.30 & \multicolumn{2}{c}{9.30} \\
\hline Slot & 7.00 & \multicolumn{2}{c}{1.00} \\
\hline Line & 1.50 & \multicolumn{2}{c}{$\begin{array}{c}1.50(\mathrm{stub})+ \\
\text { variable }\end{array}$} \\
\hline Substrate & thickness $(\mathrm{mm})$ & $\varepsilon_{\mathrm{r}}$ & $\tan \delta$ \\
\hline D1 & 7.200 & 1.000 & 0.0000 \\
\hline D2 & 0.508 & 3.380 & 0.0050 \\
\hline D3 & 2.000 & 1.067 & 0.0002 \\
\hline D4 & 0.508 & 3.380 & 0.0050 \\
\hline
\end{tabular}

resulting geometrical data are summarized in Table II. The D1 layer is air, with plastic separators only near the edge of the reflectarray. In the D2 and D4 layers, ARLON material clad with copper has been used, the patches and apertures being obtained by photo-etching. A $2 \mathrm{~mm}$ Rohacell HF51 layer has been chosen as a separator between the patches and slotted ground plane in D3. A ground plane has been included to avoid leakage of energy at the back.

The phase and amplitude of the reflection coefficient computed using the described analysis routine based on MoM in a
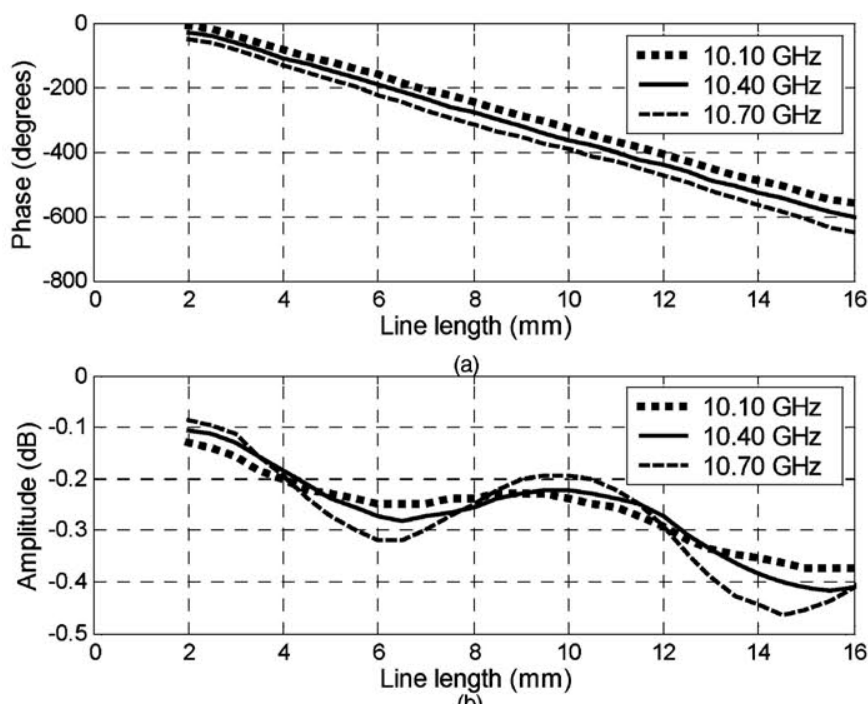

(b)

Fig. 6. (a) Phase and (b) amplitude of the reflection coefficient provided by the reflectarray element at $10.40 \mathrm{GHz}$.

periodic structure are shown in Fig. 6 as a function of the line length for central and extreme frequencies under normal incidence. The validation of this aperture-coupled reflectarray element was made by measuring the amplitude and phase of the reflection coefficient of two elements within a WR-112 [11] obtaining a satisfactory accuracy. Note that this is the reflection coefficient for the total reflected field, including the field reradiated by the patches and the one reflected by the grounded dielectric. As can be seen, the slope of the phase is very linear, and it is directly proportional to the line length, as expected for an ideal reflectarray element. Since the transmission through the apertures has been eliminated by a ground plane, the amplitude of the reflection coefficient represents the ohmic losses, which increase with the length of the line and are less than $0.4 \mathrm{~dB}$ at central frequency.

Using the previously-designed reflectarray element, the lengths of the line are adjusted, element-by-element, to match the required phase-shift. The design program is based on a zero-finding routine that iteratively calls the analysis routine until the difference between the required phase and the phase produced by the reflectarray element is less than a certain threshold. The routine takes into account the incidence angle and the polarization of the impinging wave at each element position.

Two reflectarrays have been designed, one for each linear polarization. Assuming that the feed-horn is off-set with its phase center situated at coordinates $(-175,0,390) \mathrm{mm}$, with respect the centre of the reflectarray, see Fig. 1. For the first reflectarray the delay lines are oriented along the $\mathrm{X}$ axis, which corresponds to vertical polarization; while for the second one, the delay lines are oriented along the $\mathrm{Y}$ axis corresponding to horizontal polarization. In both cases, the phase distribution shown in Fig. 4 has been achieved, with an error of less than $10^{\circ}$ in all the elements.

Figs. 7 and 8, show the photo-etching mask for the delay lines layer corresponding to each $\mathrm{V}$ and $\mathrm{H}$ orthogonal polarization, respectively. The length of the line is proportional to the phase required at each position of the reflectarray. The second and third 


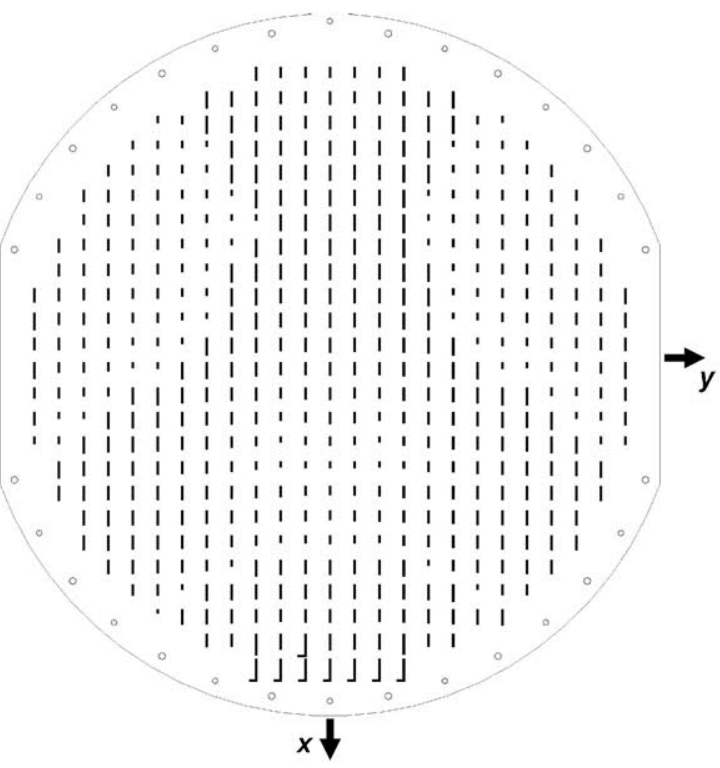

Fig. 7. Photo-etching mask for the delay lines layer corresponding to V-pol.

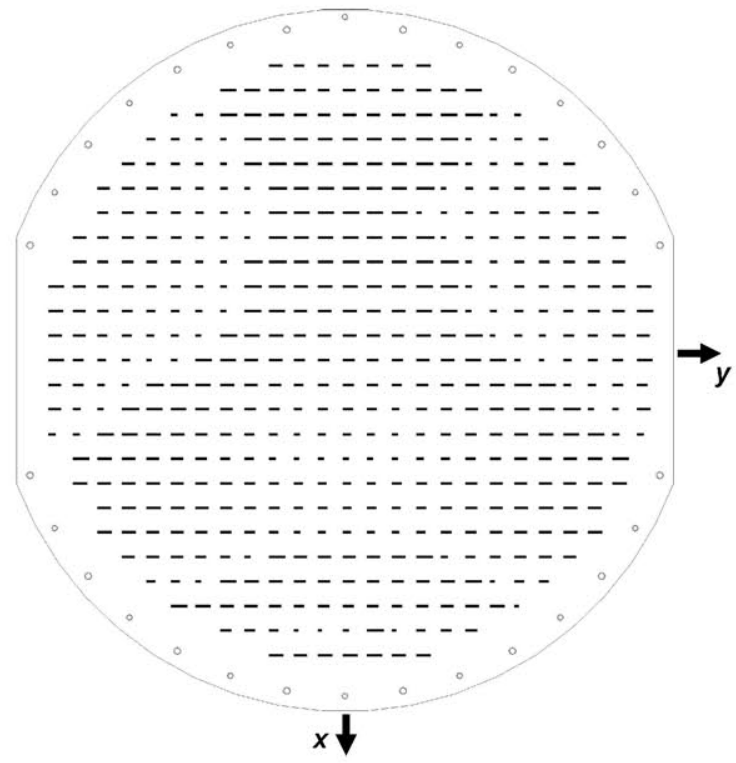

Fig. 8. Photo-etching mask for the delay lines layer corresponding to H-pol.

layers, corresponding respectively to the rectangular apertures and the square patches are common for both reflectarrays, but with an orientation difference of $90^{\circ}$ in the case of the apertures.

\section{RESULTS}

The radiating patterns have been computed for both reflectarrays to evaluate the beam-shaping behavior. The radiation pattern including co-polar and cross-polar components are computed by integrating on the reflectarray surface the total reflected plus scattered filed on each reflectarray element, which is obtained by MoM assuming local periodicity [18]. Fig. 9 shows the co-polar and cross-polar radiating patterns corresponding to the V-polarization at the central frequency. The patterns are represented in gain, taking into account spillover and ohmic losses, the radiated fields being divided by the power radiated by the

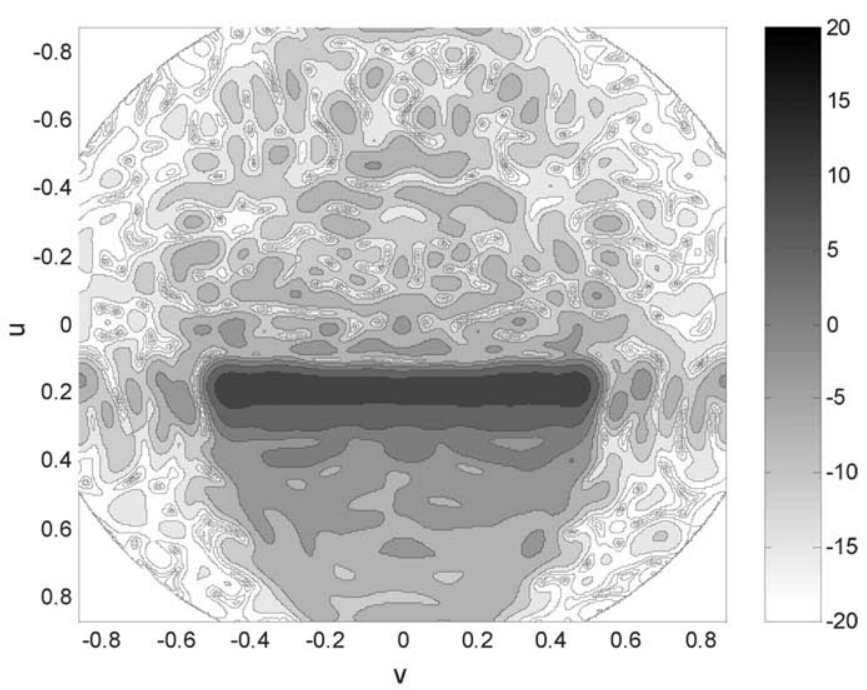

(a)

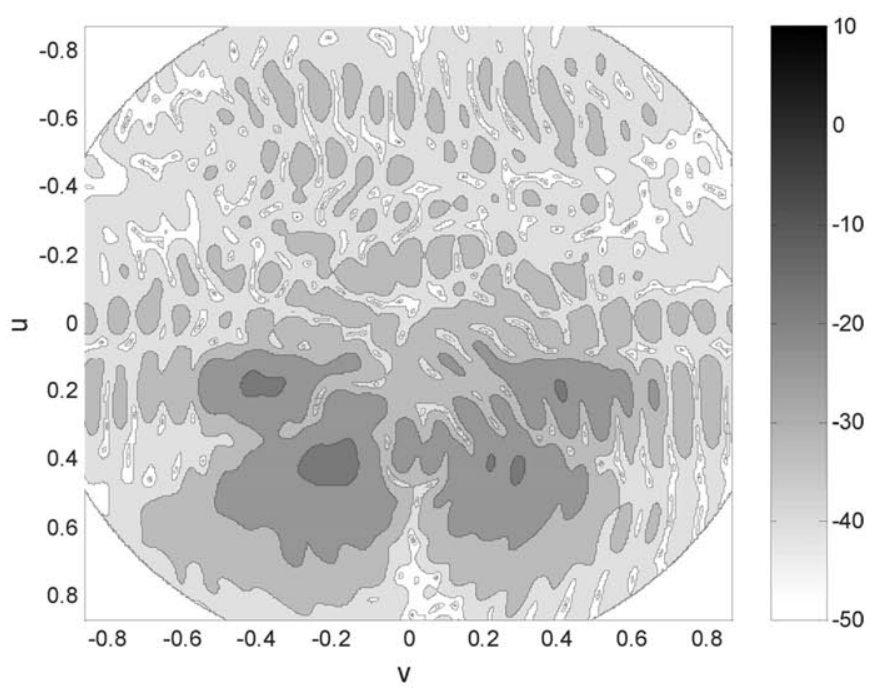

(b)

Fig. 9. Computed gain pattern for the V-pol reflectarray (a) co-polar and (b) cross-polar.

feed [18]. Fig. 10. shows the co-polar and cross-polar radiating patterns corresponding to the H-polarization. The comparison between the co-polar patterns and the specification masks are shown in Fig. 11 for both polarizations, in the elevation and azimuth planes. As can be seen, for the two polarizations, the requirements for squared cosecant and the sectorial beams are practically fulfilled. The gain allowed by the V-polarization reflectarray is at least $17.5 \mathrm{~dB}$ in the whole sector. In the case of the H-polarization reflectarray, the minimum gain obtained in the sector is $18 \mathrm{~dB}$.

From Fig. 9 it is clear that the V-pol reflectarray offers lower levels of cross-polar radiation. This reflectarray has been analyzed in the LMDS band from $10.10-10.70 \mathrm{GHz}$, and it has been manufactured and tested. The ETSI specifications are fulfilled in the entire band for the azimuth cut, with a gain of more than the $16.25 \mathrm{~dB}$ minimum requirement defined by the template. The shape of the beam is conserved in the whole band and the main differences between the extreme frequencies are only significant for levels below $10 \mathrm{~dB}$ from the maximum gain. Fig. 12 


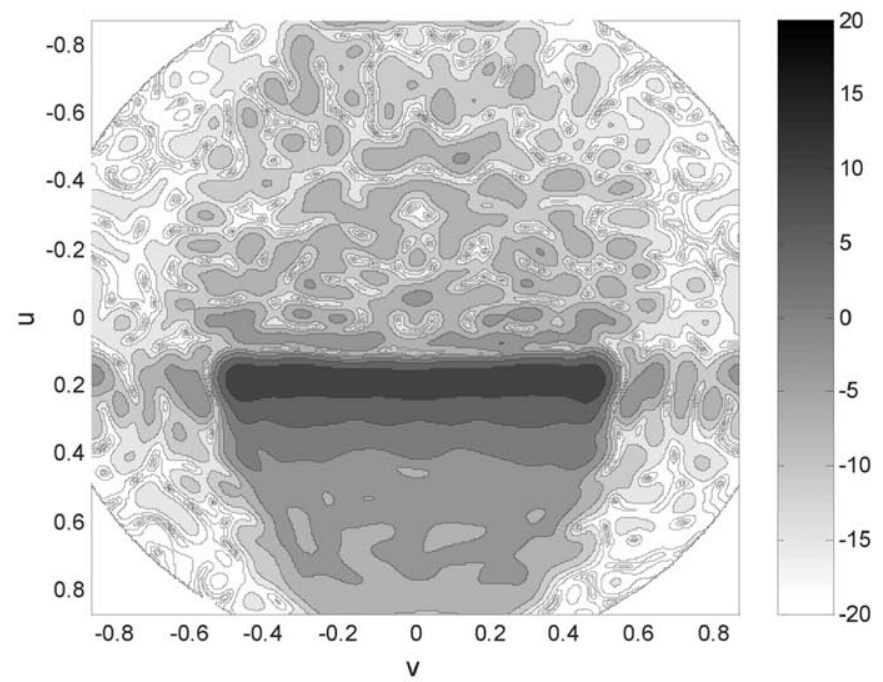

(a)

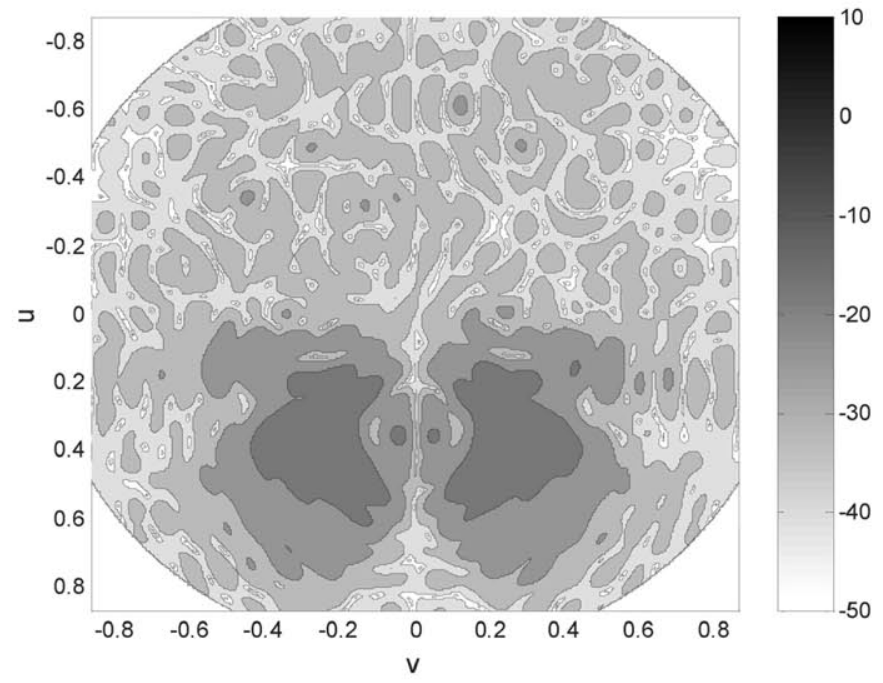

(b)

Fig. 10. Computed gain pattern for the H-pol reflectarray (a) co-polar and (b) cross-polar.

show the patterns corresponding to the elevation and azimuth planes of the V-polarization reflectarray for the LMDS band. As can be seen, the azimuth pattern is fulfilled in the $60^{\circ}$ sector. Although the LMDS only requires a $6 \%$ bandwidth, both patterns have been computed in a wide band from $9.60-11.20 \mathrm{GHz}$ which means $15 \%$ of the band, as can be seen in Fig. 13. For the elevation pattern, the shape is conserved but some nulls appear in the high extreme of the band. A fall of around $2 \mathrm{~dB}$ appears for the azimuth pattern, but the shape is conserved reasonably.

\section{BREAdBoARd Manufacture AND Test}

For the purpose of validation, a breadboard corresponding to the V-polarization has been manufactured and tested in an anechoic chamber. The slots and the delay lines have been printed on different faces of the same dielectric sheets. Each layer has been mechanically joined with a plastic screw frame to avoid changes in the electrical permittivity produced by glues. Fig. 14 shows the antenna in the test facilities.

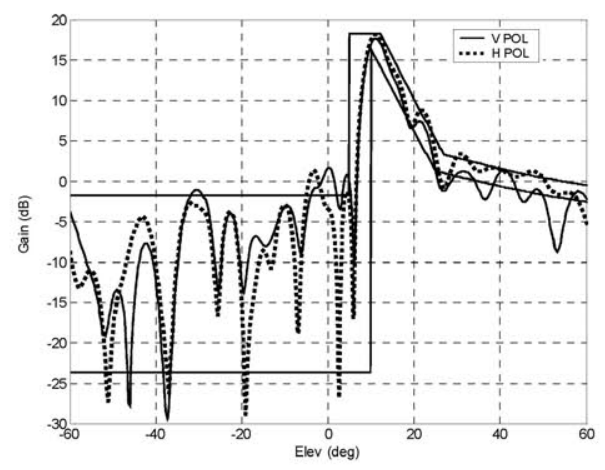

(a)

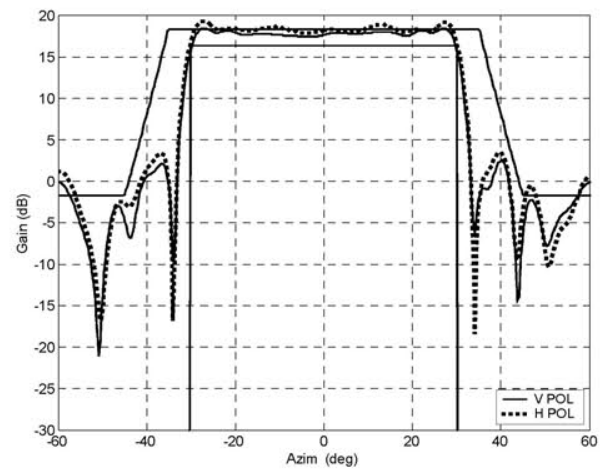

(b)

Fig. 11. Comparison between each computed linear polarization and the specification mask (a) elevation plane and (b) azimuth plane.

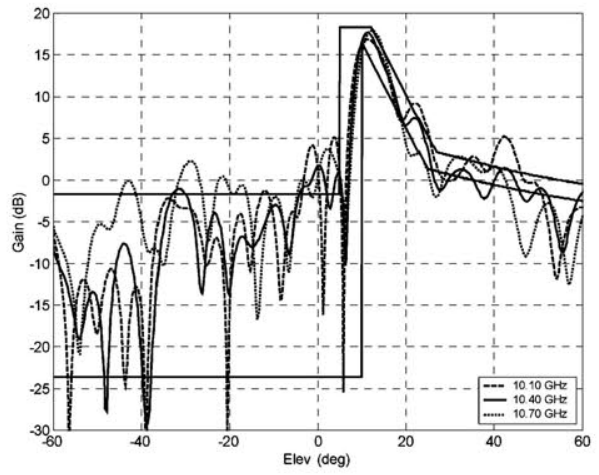

(a)

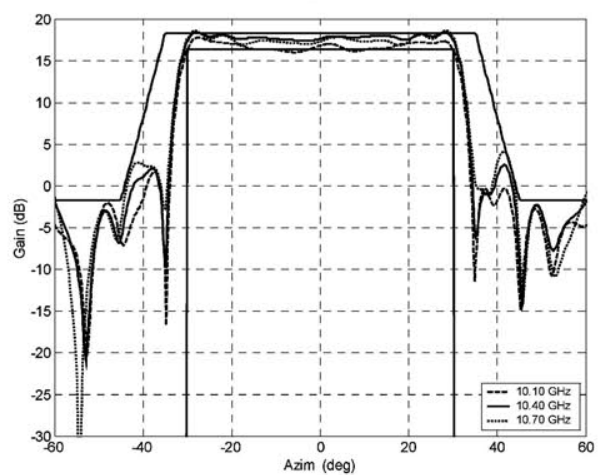

(b)

Fig. 12. Computed radiation patterns corresponding to the V-pol at the LMDS band (a) elevation plane and (b) azimuth plane.

Fig. 15 shows the measured co-polar and cross-polar radiation patterns, at the central frequency of $10.40 \mathrm{GHz}$, compared with the theoretical patterns in the elevation and azimuth 


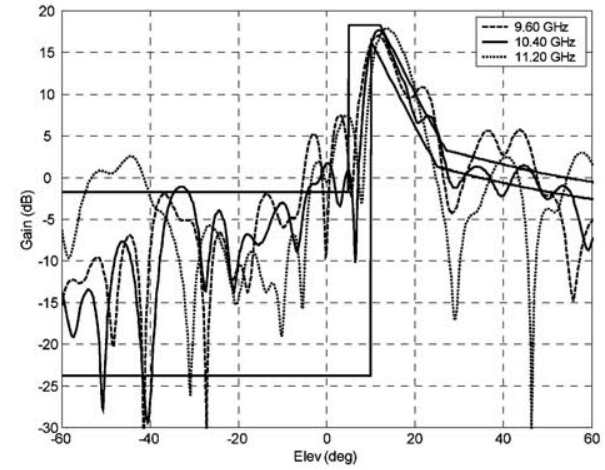

(a)

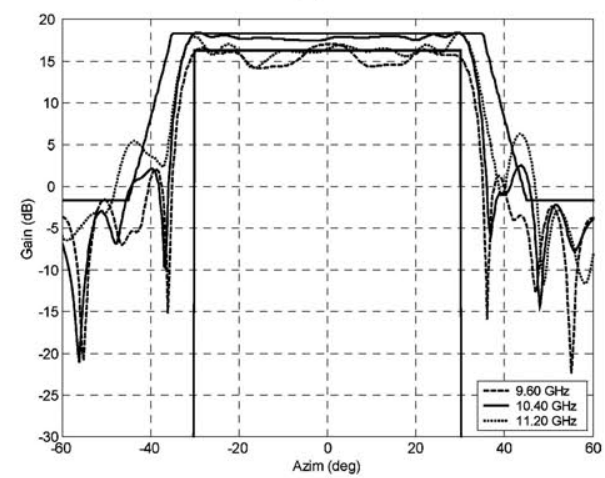

(b)

Fig. 13. Computed radiation patterns corresponding to the V-pol for a $15 \%$ bandwidth (a) elevation plane and (b) azimuth plane.

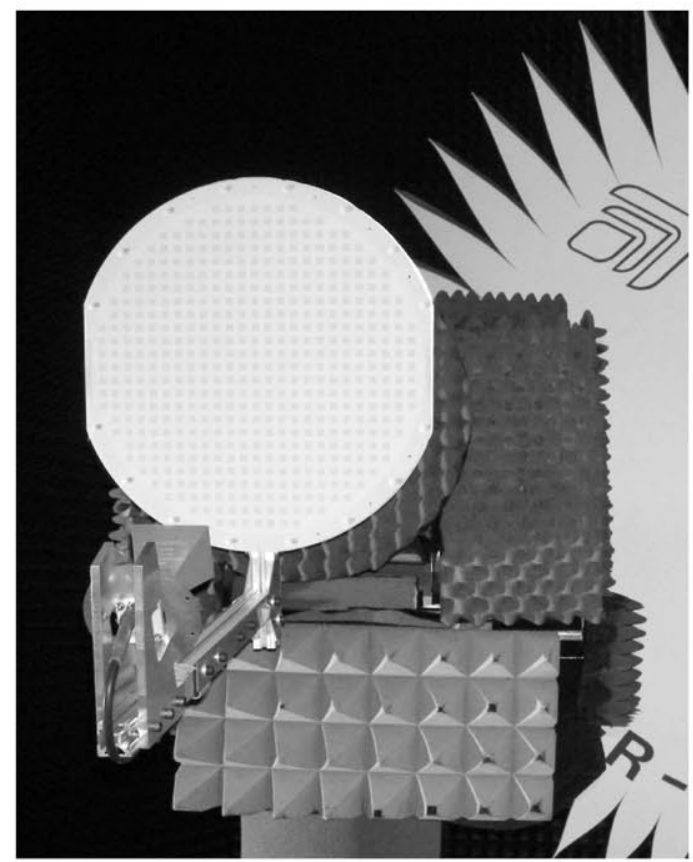

Fig. 14. Manufactured breadboard.

planes. Although the measured main lobe is practically equal to the theoretical prediction in elevation, two side lobes appear at $2^{\circ}$ and $19^{\circ}$. In the azimuth plane, Fig. $17(\mathrm{~b})$, the $60^{\circ}$ sector is fulfilled, but a with a small ripple of $1 \mathrm{~dB}$ below the specification templates shown in Fig. 2. Also a good agreement is

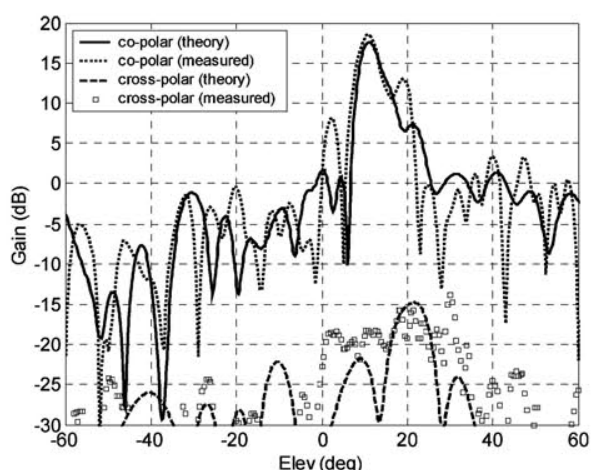

(a)

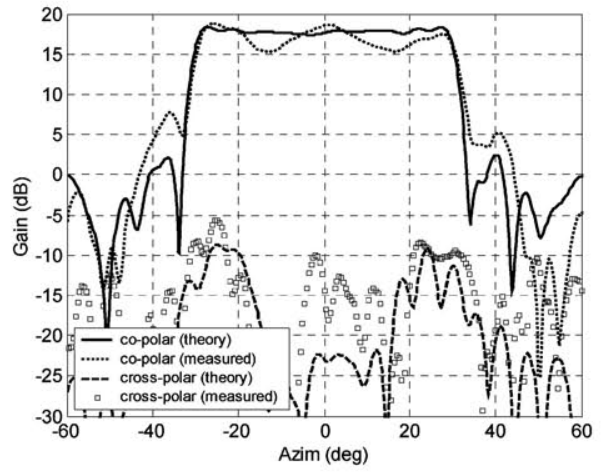

(b)

Fig. 15. Comparison between theoretical and measured radiation patterns at central frequency (a) elevation plane and (b) azimuth plane.

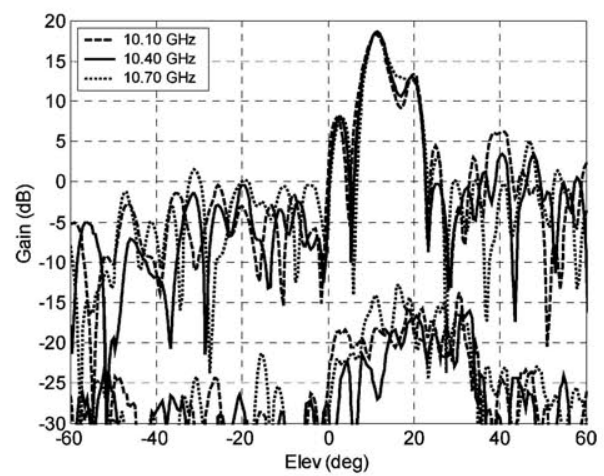

(a)

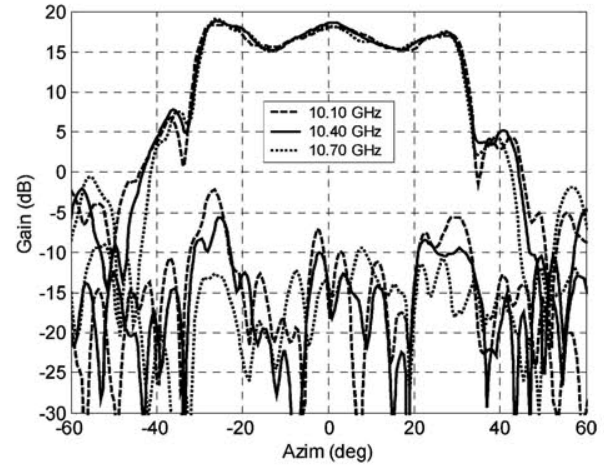

(b)

Fig. 16. Measured radiation patterns in the $10.10-10.70 \mathrm{GHz}$ band (a) elevation plane and (b) azimuth plane.

observed between simulated and measured cross-polar components. Similar measured patterns are found in the entire band from $10.10-10.70 \mathrm{GHz}$, as can be seen in Fig. 16 . 


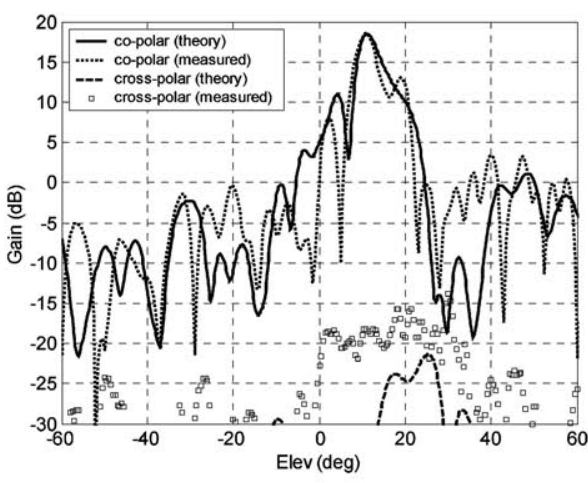

(a)

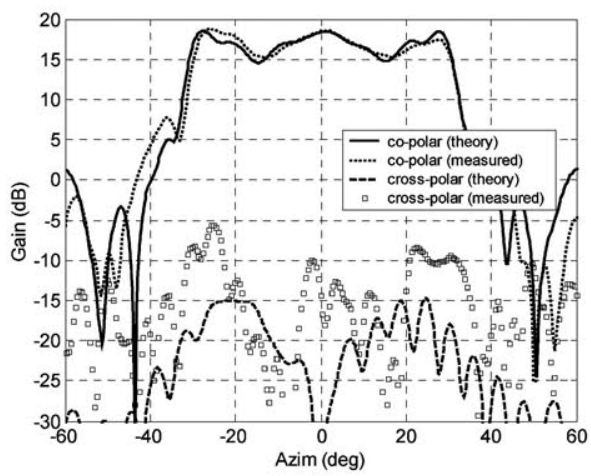

(b)

Fig. 17. Comparison between theoretical and measured radiation patterns at central frequency considering a Rohacell thickness variation of $0.2 \mathrm{~mm}$ (a) elevation plane and (b) azimuth plane.

As the aperture-coupled structure is very sensitive to thickness variations in the substrates, the small discrepancies between theoretical and measured radiation patterns are mainly produced by the thickness tolerances in the Rohacell HF51 layer. According to the manufacturer data sheets, the $2 \mathrm{~mm}$ dielectric material can vary $+0.2 \mathrm{~mm}$ from the nominal value. This variation has been evaluated in the manufactured antenna with a Vernier caliper concluding that the breadboard presents values of around $2.2 \mathrm{~mm}$ for the D3 layer. The thickness is not uniform on the whole surface, because of a small bend of the Arlon layers (D2 and D4). Some simulations have been carried out taking into account this thickness variation and a new set of radiation patterns has been obtained. Fig. 17 shows the measured co-polar and cross-polar radiation patterns, at the central frequency of $10.40 \mathrm{GHz}$, compared with the theoretical patterns when a uniform $2.2 \mathrm{~mm}$ thickness value is considered for D3. Note that the agreement between measurements and simulations has improved, showing that the errors in Rohacell thickness are producing the small pattern distortions. The remaining discrepancies can be produced by the non-uniform thickness of the D3 layer. The effects of tolerances in the nominal value of the substrate permittivity, which according with the manufacturer data sheets is less than $0.2 \%$, has already been taken into account. With this variations, there are not changes on the computed patterns for the frequency band of interest.

Although the accuracy of the technique was demonstrated in the results presented in [4] for a reflectarray made of three staked layers of varying size patches, some slight discrepancies between simulations and measurements can also be attributed to other assumptions made in the analysis tool. For example, the assumption that the field is uniform in each periodic cell, being obtained as the combination of only the fundamental TE and TM Floquet harmonic, which means that the higher order harmonics used in the analysis of the cell are ignored in the computation of the radiation pattern. In principle, the effect of the evanescent Floquet harmonics in the radiation patterns is limited to variations in the side lobe region, as can bee seen in [19]. This effect can be avoided by using higher order modes in each reflectarray cell, or by taking several samples in the cell, as described in [20].

\section{CONCLUSION}

Two reflectarrays, for vertical and horizontal linear polarization respectively, have been designed for a $60^{\circ}$ LMDS sectorial central station antenna, at $10.40 \mathrm{GHz}$ with a sectorial beam in azimuth and squared cosecant in elevation. The reflectarray configuration is based on squared patches, aperture-coupled to delay lines. This type of reflectarray provides a good accuracy in the beam shaping within the required bandwidth. A breadboard has been manufactured and tested in an anechoic chamber showing a good agreement between the theoretical and the measured radiation patterns. It has been demonstrated that the small discrepancies between theoretical and measured radiation patterns are mainly produced by the a $10 \%$ variation in the thickness of the separator between the square patches and the slotted ground plane layers. This tolerance must be reduced in order to fully accomplish the contoured-beam requirements. Although this inherent material tolerances are the main cause of discrepancy, the use of glue for bonding the layers could help to avoid air-gaps between layers and to have a uniform thickness of the Rohacell layers.

Finally, the proposed aperture-coupled reflectarray can incorporate switches or controllable phase-shifters in the delay lines in order to achieve a reconfiguration of the beam.

\section{REFERENCES}

[1] European Standard (Telecommunication Series), Fixed Radio Systems; Point-to-Multipoint Antennas; Antennas for Point-to-Multipoint Fixed Radio Systems in the $3 \mathrm{GHz}$ to $11 \mathrm{GHz}$ Band 2005, ETSI EN 302085 v1.2.3.

[2] Harmonised Radio Frequency Channel Arrangements for Digital Terrestrial Fixed Systems Operating in the Band 10.0-10.68 GHz Rome, 1996, CEPT/ERC/REC 12-05 E.

[3] J. A. Encinar and A. Zornoza, "Three-layer printed reflectarrays for contoured beam space applications," IEEE Trans. Antennas Propag., vol. 52, no. 5, p. 11381148 , May 2004.

[4] J. A. Encinar, K. S. Datashvili, J. A. Zornoza, M. Arrebola, M. SierraCastañer, J. L. Besada-Sanmartin, H. Baier, and H. Legay, "Dual-polarization dual-coverage reflectarray for space applications," IEEE Trans. Antennas Propag., vol. 54, no. 10, pp. 2827-2837, Oct. 2006.

[5] M. Arrebola, J. A. Encinar, and M. Barba, "Multifed printed reflectarray with three simultaneous shaped beams for LMDS central station antenna," IEEE Trans. Antennas Propag., vol. 56, no. 6, pp. 1518-1527, Jun. 2008.

[6] A. Bhattacharyya, "Slot-coupled patch reflect array element for enhanced gain-bandwitdh performance," U.S. 6,388,620, 2000.

[7] M. E. Bialkowski and H. J. Song, "Dual linearly polarized reflectarray using aperture coupled microstrip patches," in Proc. Antennas and Propagation Society Int. Symp., Jul. 2001, pp. 486-489.

[8] M. Barba, E. Carrasco, J. E. Page, and J. A. Encinar, "Electronic controllable reflectarray elements in X band," Frequenz, J. RF-Eng. Telecommun., vol. 61, pp. 203-206, Sep.-Oct. 2007.

[9] B. Mencagli, R. Vincenti, L. Marcaccioli, and R. Sorrentino, "Design of large mm-wave beam-scanning reflectarrays," presented at the 35th Eur. Microwave Conf., París, France, Oct. 2005. 
[10] J. Huang, "Bandwidth study of microstrip reflectarray and a novel phased reflectarray concept," in Antennas and Propag. Society Int. Symp. Digest, Jun. 1995, vol. 1, pp. 582-585.

[11] E. Carrasco, M. Barba, and J. A. Encinar, "Aperture-coupled reflectarray element with wide range of phase delay," Electron.Lett., vol. 42, pp. 667-668, Jun. 2006.

[12] E. Carrasco, J. A. Encinar, and M. Barba, "Bandwidth improvement in large reflectarrays by using true-time delay," IEEE Trans. Antennas Propag., vol. 56, no. 8, pp. 2496-2503, Aug. 2008.

[13] E. Carrasco, M. Barba, and J. A. Encinar, "Reflectarray element based on aperture-coupled patches with slots and lines of variable length," IEEE Trans. Antennas Propag., vol. 55, pp. 820-825, Mar. 2007.

[14] C. H. Wan and J. A. Encinar, "Efficient computation of generalized scattering matrix for analyzing multilayer periodic structures," IEEE Trans. Antennas Propag., vol. 43, no. 11, pp. 1233-1242, 1995.

[15] O. M. Bucci, A. Capozzoli, G. D'Elia, and S. Musto, "A new approach to the power pattern synthesis of reflectarrays," in Proc. URSI EMTS Symp., Pisa, Italy, May 2004, pp. 1053-1055.

[16] A. Chakraborty, B. N. Das, and G. S. Sanyal, "Beam shaping using nonlinear phase distribution in a uniformly spaced array," IEEE Trans. Antennas Propag., vol. 30, pp. 1031-1034, Sep. 1982.

[17] O. M. Bucci, G. Franceschetti, G. Mazzarella, and G. Panariello, "Intersection approach to array pattern synthesis," Proc. Inst. Elect. Eng. Microw., Antennas Propag., vol. 137, no. 6, pp. 349-357, Dec. 1990.

[18] J. Huang and J. A. Encinar, Reflectarrays Antennas. New York: Wiley, 2007, pp. 68-74.

[19] J. A. Encinar, "Analysis and CAD techniques for periodic leaky-wave printed antennas: Numerical and experimental results," Int. J. Microw. Millimeter-Wave Comput.-Aided Eng., vol. 4, no. 1, pp. 88-99, Jan. 1994.

[20] M. Arrebola, Y. Álvarez, J. A. Encinar, and F. Las-Heras, "Accurate analysis of printed reflectarrays considering the near field of the primary feed," IET Microw., Antennas Propag., to be published.

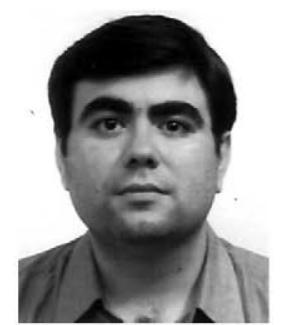

Manuel Arrebola (S'99-M'07) was born in Lucena, Spain. He received the Ingeniero de Telecomunicacion degree from Universidad de Malaga (UMA), Malaga, Spain, in 2002, and he is currently working toward the Ph.D. degree at Universidad Politecnica de Madrid (UPM), Madrid, Spain.

From 2003 to 2007 , he was with the Electromagnetism and Circuit Theory Department, UPM, as a Research Assistant. From August to December 2005, he was with the Microwave Techniques Department, Universität Ulm, Ulm, Germany, as a Visiting Scholar. In December 2007, he joined the Electrical Engineering Department, Universidad de Oviedo, Gijon, Spain, where he is an Assistant Professor. His current research interests include analysis and design techniques of printed reflectarray antennas.

Mr. Arrebola was corecipient of the IEEE Antennas and Propagation Society 2007 S. A. Schelkunoff Prize Paper Award.

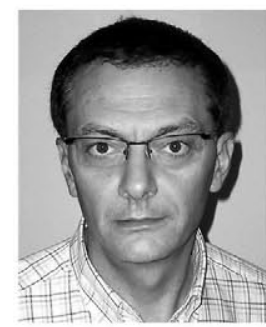

Jose A. Encinar (S'81-M'86) was born in Madrid, Spain. He received the Electrical Engineer and Ph.D. degrees, both from Universidad Politecnica de Madrid (UPM), in 1979 and 1985, respectively.

Since January 1980 , has been with the Applied Electromagnetism and Microwaves Group, UPM, as a Teaching and Research Assistant from 1980 to 1982, as an Assistant Professor from 1983 to 1986 , and as Associate Professor from 1986 to 1991 . From February to October 1987, he was with Polytechnic University, Brooklyn, NY, as a Postdoctoral Fellow of the NATO Science Program. Since 1991, he is a Professor of the the Electromagnetism and Circuit Theory Department, UPM. In 1996, he was with the Laboratory of Electromagnetics and Acoustics, Ecole Polytechnique Federale de Lausanne (EPFL), Switzerland, as a Visiting Professor. His research interests include numerical techniques for the analysis of multilayer periodic structures, design of frequency selective surfaces, printed arrays and reflectarrays. He has published more than 100 journal and conference papers, and is the holder of three patents on array and reflectarray antennas.

Prof. Encinar was a corecipient of the $2005 \mathrm{H}$. A. Wheeler Applications Prize Paper Award and the 2007 S. A. Schelkunoff Transactions Prize Paper Award, given by IEEE Antennas and Propagation Society.

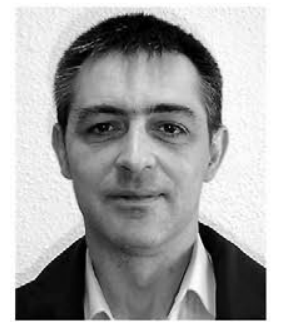

Mariano Barba was born in Murcia, Spain. He received the Ingeniero de Telecomunicacion and $\mathrm{Ph} . \mathrm{D}$. degrees from the Universidad Politecnica de Madrid, Madrid (UPM), Spain, in 1990 and 1996, respectively.

From 1991 to 1994, he was with the Departamento de Electromagnetismo y Teoria de Circuitos, UPM, as a Postgraduate Researcher. During this time he developed numerical methods and algorithms to characterize and design microwave passive devices. From 1994 to 2003, he has been involved in the R\&D departments of several companies and institutions developing antennas and microwave circuits for space and terrestrial communication applications. In 2003, he joined to the Departamento de Electromagnetismo y Teoria de Circuitos, UPM, as an Associate Professor. His research interests include the analysis, characterization and design of antenna and microwave circuits. He is currently involved in the development and design of multibeam and reconfigurable antennas. 